Recent Insights into the Physics of the Sun and Heliosphere:

Highlights from SOHO and Other Space Missions

IAU Symposium, Vol. 203, 2001

P. Brekke, B. Fleck, and J. B. Gurman eds.

\title{
Wave Induced Synergetic Electron Acceleration in Inhomogeneous Solar Plasmas
}

\author{
M. P. Leubner \\ Institute for Theoretical Physics, University of Innsbruck, A-6020 \\ Innsbruck, Austria
}

\begin{abstract}
Suprathermal electron populations are generated efficiently in uniform space plasmas by resonant Landau interaction with broadband Alfvén wave spectra. Based on a simulation of the particle acceleration in response to the wave energy input within the Fokker-Planck formalism, details of the time evolution of the velocity space distribution towards commonly observed solar wind power law spectra are discussed. Furthermore, it is shown that the diffusion properties and consequently the particle energization change significantly in astrophysical environments with magnetic field and density gradients, since synergetic effects become dominant. Electrons are accelerated resonantly out of the bulk of the distribution such that they interact again with a wave packet of higher phase velocity, leading to a multi-stage energization. Hence, a unique acceleration mechanism can be achieved without postulating pre-acceleration by ad hoc mechanisms. Depending on the density and magnetic field profile, significant enhancement of energetic particles as well as stagnation in a saturated stage of suprathermal, non-Maxwellian velocity space distributions is possible. The importance of synergetic acceleration in complex solar flare structures and for solar wind heating mechanisms is discussed in relation to spacecraft observations.
\end{abstract}

Particle acceleration in the heliosphere is confirmed by a large variety of spacecraft observations as provided by Helios, Voyager, Wind or Soho experiments where energetic electrons, protons or heavy ions are found as ubiquitous property of solar wind-coronal conditions. We have to consider particle energization as a universal phenomenon ranging from solar flare conditions and the upper chromosphere throughout into the outer interplanetary space. Besides DC-electric fields (Anastasiadis, Vlahos, \& Georgoulis 1997) or potential drops at magnetic field line reconnection regions wave acceleration due to resonant interaction is a highly significant mechanism for the generation of suprathermal tails in the velocity space distributions of uniform solar plasmas (Leubner, 2000).

Alternatively, non-uniform magnetic fields and density gradients significantly enhance particle energization due to synergetic effects, resulting in a multi-stage acceleration process. Consider an Alfvén wave spectrum in an inhomogeneous solar plasma and let us simulate the resonant particle acceleration in response to the wave energy input in terms of a Fokker-Planck approach. Gradients are able to generate multiple wave packets of increasing phase velocities. Hence, electrons are accelerated out of the bulk of the distribution function such that they become again resonant with a wave packet of higher phase speed. This 

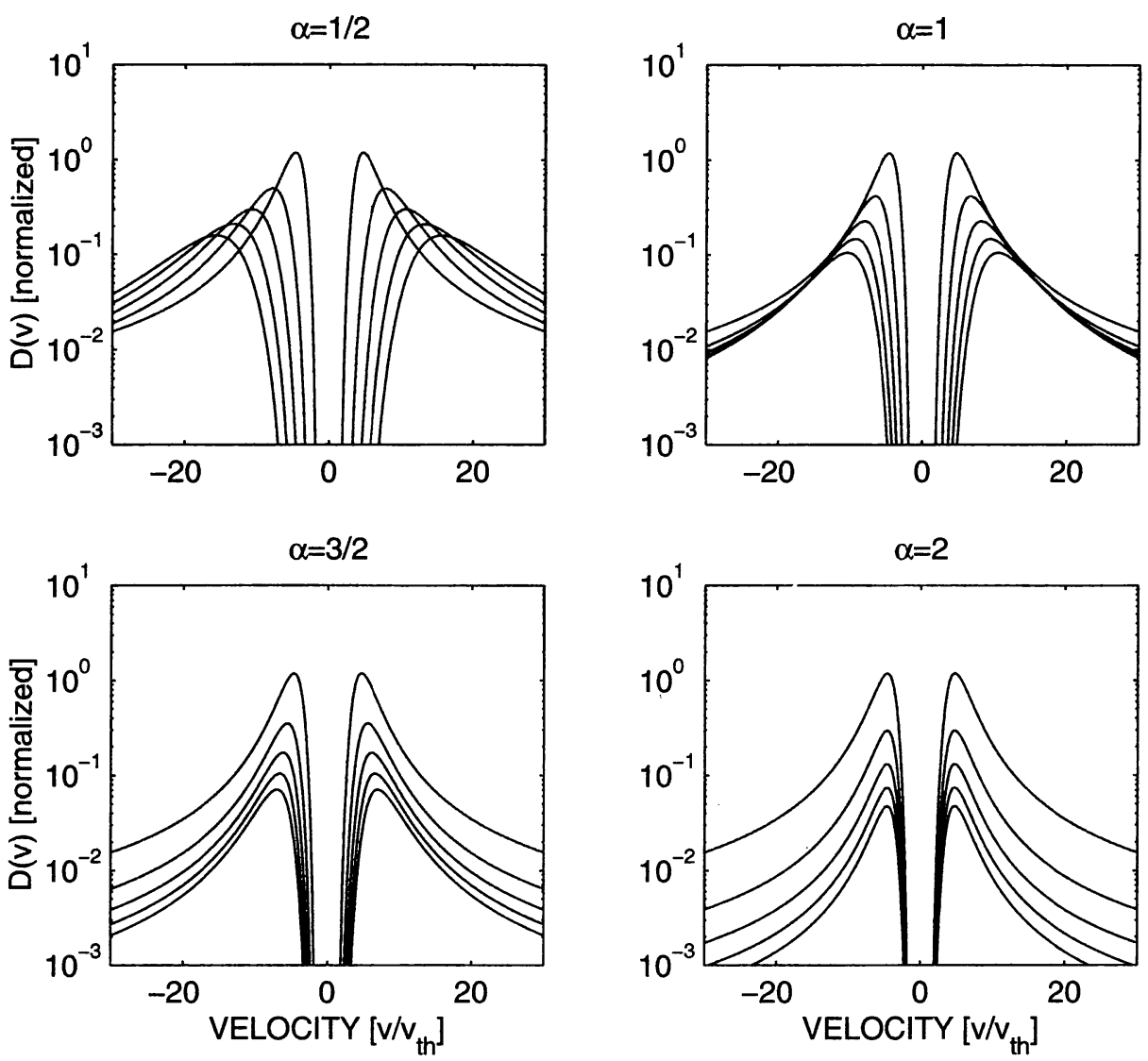

Figure 1. Snapshots of the changes of the diffusion properties in velocity space due to magnetic field gradients and density variations according to the relation $B^{\alpha}=$ const $* N$.

process yields a highly efficient particle energization without proposing preacceleration mechanisms as cascading fast mode waves (Miller, LaRosa, \& Moore 1996) Hence, the magnetic field and density gradients act as catalyst.

Consider the general equation for the collective time evolution of the distribution function in response to collisions and resonant Landau interaction

$$
\frac{\partial f}{\partial t}=\frac{\partial}{\partial v} \nu(v)\left[v f+v_{t h}^{2} \frac{\partial f}{\partial v}\right]+\frac{\partial}{\partial v} D(v) \frac{\partial f}{\partial v}
$$

where $\nu(v)$ and $D(v)$ denote the velocity dependent collision and diffusion operators, respectively. The first term on the right hand side of equation (1) restores the distribution function to a Maxwellian. Next, define the quasi-linear, one dimensional diffusion operator due to Landau wave-particle interaction by 
(a)

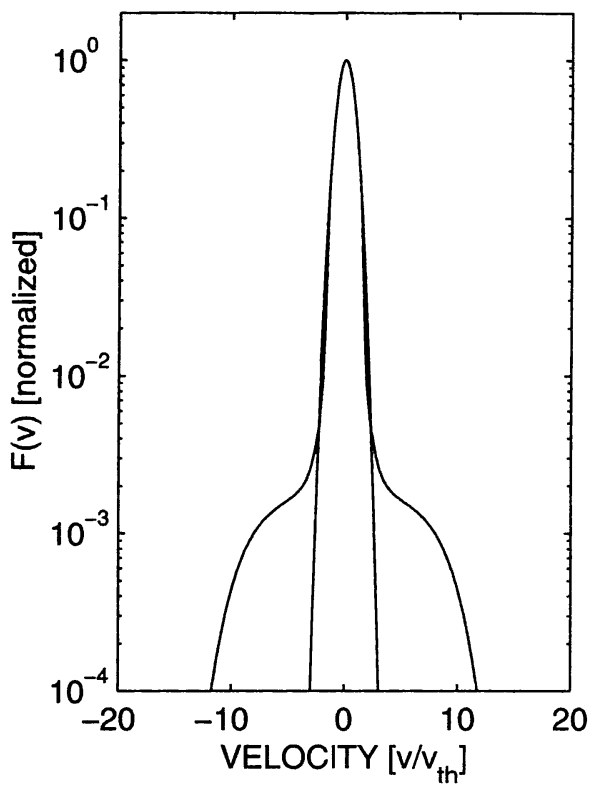

(b)

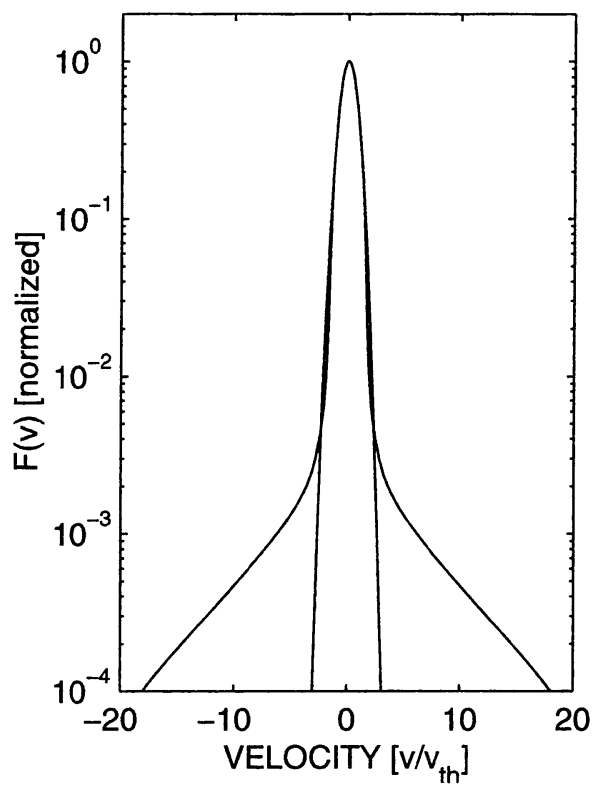

Figure 2. (a) Snapshot of the time evolution of the distribution function due to synergetic effects. (b) Saturated stage showing significant particle energization. Velocities are normalized to the thermal speed.

$$
D(v)=\left(\frac{e}{m} \cos \Theta\right)^{2} \int \frac{d \Omega d K}{2 \pi} S_{E}(K, \Omega) \pi \delta(\Omega-K v \cos \Theta)
$$

where $S(K, \Omega)$ is the spectral energy density of the wave turbulence and the Dirac term describes the wave-particle resonance. The $\cos \theta$ dependence is a consequence of the wave parallel electric field component. After adopting a broadband Alfvén wave spectrum and introducing the dispersion relation for the shear kinetic case the time evolution of the distribution function due to resonant wave-particle interaction can be simulated numerically.

It turns out that the magnetic field and density variations predominantly determine the resonance properties of the interactions, see Fig. 1 whereas temperature variations are of minor importance. Hence, $T=$ const. is assumed and a solar plasma obeying $B^{\alpha}=$ const. $* N$ as model for the field line convergence is studied. Fig. 2 presents an example of the resulting efficient electron energization from a starting Maxwellian and solar flare conditions.

\section{References}

Anastasiadis, A., Vlahos, L., Georgoulis, M. K. 1997, ApJ, 489, 367

Leubner, M. P. 2000, Planet. Space Sci., 48, 133

Miller, J. A., LaRosa, T. N., \& Moore, R. L. 1996, ApJ, 461, 445 